\title{
The global landscape of neoadjuvant and adjuvant anti-PD-1/PD-L1 clinical trials
}

\author{
Dawei Wu ${ }^{1 \dagger}$, Huiyao Huang ${ }^{1 \dagger}$, Minghui Zhang ${ }^{2 \dagger}$, Ziwei $\mathrm{Li}^{1,3}$, Shuhang Wang ${ }^{1}$, Yue Yu ${ }^{1}$, Yuan Fang ${ }^{1}$, Ning Jiang ${ }^{1}$, \\ Huilei Miao ${ }^{1}$, Peiwen Ma ${ }^{1}$, Yu Tang ${ }^{1}$ and Ning $\mathrm{Li}^{i^{*}}$ (D)
}

\begin{abstract}
The neoadjuvant and adjuvant anti-PD-1/PD-L1 treatment has been increasingly noticed. To summarize the global landscape of these clinical trials will provide essential data for all the stakeholders of drug development. Based on the Trialtrove database, a total of 668 clinical trials initiated by the end of 2020 were retrospectively analyzed. We found that a rising capability of global neoadjuvant and adjuvant anti-PD-1/PD-L1 clinical development has been achieved. High prevalent cancer types were extensively studied though the priorities in China and the United States were different. However, a lack of phase III trials and industry-sponsored trials was addressed. The confirmatory neoadjuvant trials were particularly insufficient, and the combination strategy mainly focused on chemotherapy. Thus, more public funding and accelerated regulatory strategies are needed in this field. Efforts should be made to confirm the benefit of neoadjuvant treatment and explore novel combination strategies.
\end{abstract}

Keywords: Neoadjuvant, Adjuvant, Anti-PD-1/PD-L1 treatment, Clinical trial

\section{To the editor}

Anti-PD-1/PD-L1 treatment is now the standard of care for many cancer types worldwide $[1,2]$. Based on the feasibility of checkpoint blockade in the earlier stage of cancer [3-6], neoadjuvant and adjuvant immunotherapy has attracted more attention, especially neoadjuvant settings [3]. However, the evidence on the global panorama of this field is limited. Most of the relevant studies focused on specific cancer types, such as melanoma [7, 8], without the time trend and geographic information. Therefore, we will give a comprehensive analysis of the current pipeline, thus providing essential supportive data for industry, clinical institutions and regulatory authorities.

\footnotetext{
*Correspondence: lining@cicams.ac.cn

tDawei Wu, Huiyao Huang and Minghui Zhang have contributed equally to this work

${ }^{1}$ Clinical Trials Center, National Cancer Center/National Clinical Research Center for Cancer/Cancer Hospital, Chinese Academy of Medical Sciences and Peking Union Medical College, Beijing, China

Full list of author information is available at the end of the article
}

Until December 31, 2020, a total of 668 eligible neoadjuvant and adjuvant anti-PD-1/PD-L1 clinical trials were retrieved from the Trialtrove database [9] (Additional file 1: Fig. S1). The annual number of trials showed an upward trend $(\mathrm{F}=25.5, p=0.001)$, with a compound annual growth rate of $73.6 \%$. Phase II trials accounted for the highest proportion $(427,63.9 \%)$, followed by phase I $(161,24.1 \%)$ and phase III $(80,12.0 \%)$ (Fig. 1). There were $433(64.8 \%)$ investigator-initiated trials (IITs) and 235 (35.2\%) industry-sponsored trials (ISTs). The IIT was the major type of clinical trials hosted by the United States (149/283, 52.7\%) and China (201/216, 93.1\%) (Additional file 1: Fig. S2).

Despite the significant increase in trial number, the lack of phase III trials and ISTs suggested that neoadjuvant and adjuvant anti-PD-1/PD-L1 treatment was still at its early exploratory stage. In addition, due to the need for a multidisciplinary team and a prolonged follow-up time, only large pharmaceutical enterprises have the ability to carry out confirmatory registration trials. The country distribution of ISTs is consistent with that of top pharma 
300

249

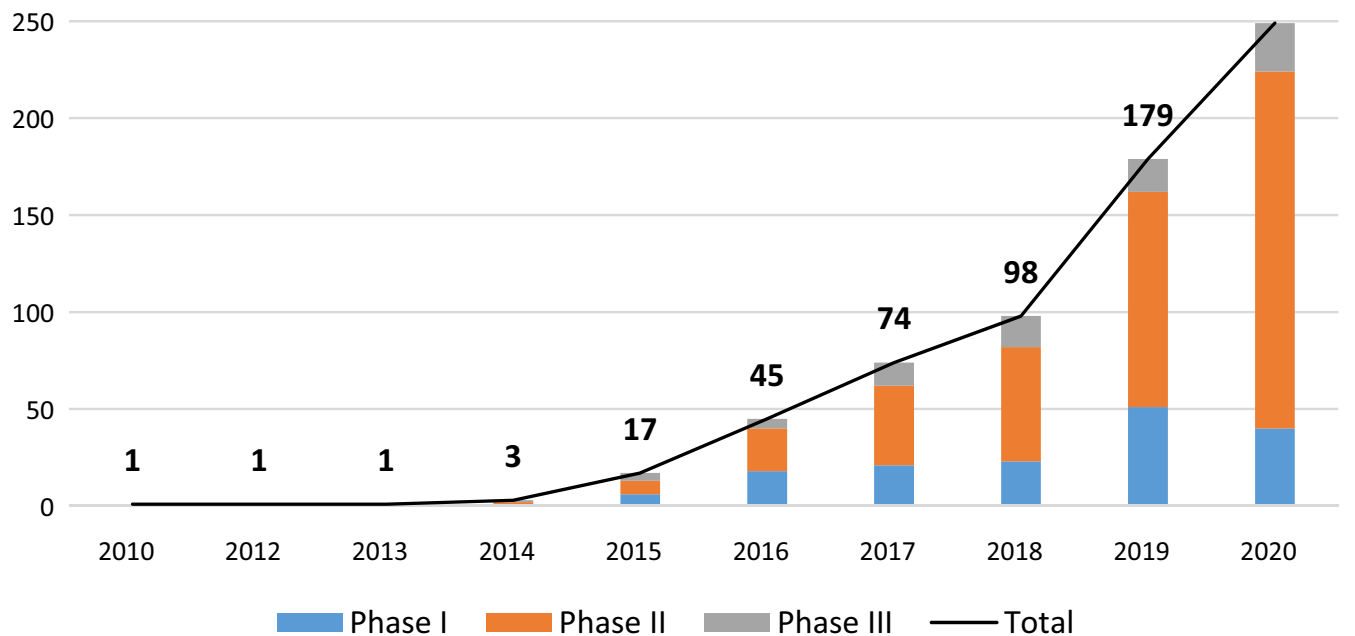

Fig. 1 Annual numbers of initiated neoadjuvant and adjuvant anti-PD-1/PD-L1 trials worldwide, overall and by study phase. The compound annual growth rates of overall, phase I, phase II and phase III trials were $73.6 \%, 44.6 \%, 91.9 \%$ and $71.0 \%$, respectively

companies (Additional file 1: Fig. S2). In the future, more experts for multidisciplinary treatment are needed by industries. Policymakers should consider more funding in this field, and formulate accelerated regulatory strategies for the review and approval process, with the application of novel surrogate endpoints, such as pathological response indicators $[5,10]$.

A total of 24 cancer types were identified in the analysis. Non-small-cell lung cancer (NSCLC), breast cancer (70, 10.5\%), esophageal cancer $(60,9.0 \%)$ and melanoma (60, 9.0\%) were the most common cancers. There were huge differences in the cancer type distribution of clinical trials hosted by the United States and China based on the different clinical needs. Melanoma, breast cancer and urothelial carcinoma that were focused on in the United States, had a relatively high proportion of localized stage at diagnosis [11]. Clinical trials of China mainly targeted esophageal cancer, gastric cancer and hepatocellular carcinoma (HCC), which were highly prevalent and associated with different causes compared to western countries [12] (Table 1).

For the treatment mode, on the basis of feasibility including assessing the effect via biopsy of surgical specimen, reducing tumor size before surgery and inducing greater T-cell expansion [3, 6], neoadjuvant mode (544, $81.4 \%)$ has attracted more attention. The time trend (Additional file 1: Fig. S3) and geographic distribution (Additional file 1: Fig. S4) of neoadjuvant trials were consistent with that of overall trials. However, among phase III trials, the proportion of neoadjuvant trials $(42 / 80$,
52.5\%) was not that large (Additional file 1: Table S1). More confirmatory evidence is needed to illustrate the optimal sequence of immunotherapy and surgery, for whether preoperative treatment can bring long-term survival benefits.

Most of the clinical trials were testing combination regimens $(554,82.9 \%)$, and chemotherapy was the most commonly used combination partner in both neoadjuvant $(286 / 455,62.9 \%)$ and adjuvant $(89 / 167,53.3 \%)$ phases (Additional file 1: Table S2). Adding PD-1/PD-L1 mAbs to standard neoadjuvant or adjuvant chemotherapy of major cancers was found to be a regular design of combination trials, especially phase III trials (Additional file 1: Table S1). However, in the neoadjuvant phase, combination strategies designed to recruit more immune cells into the tumor, such as immuno-oncology (IO) agents, may be more promising [3-6]. How to expand combination strategies and break through the framework of the traditional neoadjuvant chemotherapy remains to be studied in depth.

In conclusion, the neoadjuvant and adjuvant antiPD-1/PD-L1 clinical trials have developed rapidly worldwide. High prevalent cancer types with clinical needs have been concerned though the priorities in China and the United States were different. But the clinical development of this field is still at early stage. There are challenges including how to balance the huge cost of clinical operation by public funding and accelerated regulatory strategies, and how to confirm the benefit of neoadjuvant treatment and optimize combination strategies. 
Table 1 Cancer type distribution of neoadjuvant and adjuvant anti-PD-1/PD-L1 trials and the comparison among host country

\begin{tabular}{|c|c|c|c|c|}
\hline \multirow[t]{2}{*}{ Cancer type } & \multicolumn{4}{|c|}{ Host country } \\
\hline & China & United States & Rest of world & Total \\
\hline Non-small-cell lung cancer & 39 & 26 & 35 & 100 \\
\hline Breast cancer & 10 & 30 & 30 & 70 \\
\hline Esophageal cancer & 46 & 8 & 6 & 60 \\
\hline Melanoma & 10 & 41 & 9 & 60 \\
\hline Urothelial carcinoma & 10 & 29 & 20 & 59 \\
\hline Head and neck squamous cell carcinoma & 13 & 29 & 14 & 56 \\
\hline Colorectal cancer & 16 & 13 & 16 & 45 \\
\hline Gastric cancer & 25 & 11 & 8 & 44 \\
\hline Hepatocellular carcinoma & 23 & 7 & 6 & 36 \\
\hline Renal cancer & 5 & 10 & 4 & 19 \\
\hline Pancreatic cancer & 1 & 14 & 4 & 19 \\
\hline Glioma & 3 & 10 & 2 & 15 \\
\hline Soft tissue sarcoma & 4 & 6 & 2 & 12 \\
\hline Cutaneous squamous cell carcinoma & 1 & 8 & 2 & 11 \\
\hline Mesothelioma & 0 & 11 & 0 & 11 \\
\hline Ovarian cancer & 0 & 5 & 5 & 10 \\
\hline Solid tumor & 0 & 6 & 1 & 7 \\
\hline Biliary tract cancer & 4 & 1 & 1 & 6 \\
\hline Prostate cancer & 0 & 5 & 1 & 6 \\
\hline Cervical cancer & 2 & 2 & 0 & 4 \\
\hline Endometrial carcinoma & 0 & 4 & 0 & 4 \\
\hline Merkel cell carcinoma & 0 & 3 & 1 & 4 \\
\hline Thyroid cancer & 2 & 1 & 0 & 3 \\
\hline Small-cell lung cancer & 2 & 0 & 0 & 2 \\
\hline Pancreatic cancer and colorectal cancer & 0 & 1 & 0 & 1 \\
\hline Gastric cancer and colorectal cancer & 0 & 0 & 1 & 1 \\
\hline NSCLC and HCC & 0 & 1 & 0 & 1 \\
\hline NSCLC and Gastric cancer & 0 & 0 & 1 & 1 \\
\hline Colorectal cancer and pancreatic cancer & 0 & 1 & 0 & 1 \\
\hline Total & 216 & 283 & 169 & 668 \\
\hline
\end{tabular}

\section{Abbreviations}

IIT: Investigator-initiated trial; IST: Industry-sponsored trial; NSCLC: Non-smallcell lung cancer; HCC: Hepatocellular carcinoma; IO: Immuno-oncology.

\section{Supplementary Information}

The online version contains supplementary material available at https://doi. org/10.1186/s13045-022-01227-1.

Additional file 1: Data processing details and additional results

\section{Acknowledgements}

Not applicable.

\section{Authors' contributions}

WDW, HHY and ZMH contributed to framework planning and draft writing, as well as data quality control, analysis and interpretation. LZW participated in data quality control and interpretation. WSH, YY, FY, JN, MHL, MPW and TY participated in framework planning and contributed to data interpretation. NL led the overall framework planning and data interpretation. All authors read and approved the final manuscript.

\section{Funding}

This work was supported by the Construction of Clinical Research Ward in Beijing (BCRW202003).

\section{Availability of data and materials}

All the source data in this work are based on the Trialtrove database, with clinical trial details derived from clinical trial publicity platforms. The datasets used and analyzed during the study are available from the corresponding author on reasonable request.

\section{Declarations}

Ethics approval and consent to participate

Not applicable. 


\section{Consent for publication}

The content of the manuscript has not been previously published and is not under consideration for publication elsewhere.

\section{Competing interests}

The authors declare that they have no competing interests.

\section{Author details}

${ }^{1}$ Clinical Trials Center, National Cancer Center/National Clinical Research Center for Cancer/Cancer Hospital, Chinese Academy of Medical Sciences and Peking Union Medical College, Beijing, China. ${ }^{2}$ Department of Medical Oncology, Harbin Medical University Cancer Hospital, Harbin, China. ${ }^{3}$ Department of Basic Medicine and Clinical Pharmacy, China Pharmaceutical University, Nanjing, China.

Received: 18 November 2021 Accepted: 10 January 2022 Published online: 08 February 2022

\section{References}

1. Yu JX, Hodge JP, Oliva C, Neftelinov ST, Hubbard-Lucey VM, Tang J. Trends in clinical development for PD-1/PD-L1 inhibitors. Nat Rev Drug Discov. 2020;19(3):163-4

2. Wu DW, Huang HY, Tang Y, et al. Clinical development of immuno-oncology in China. Lancet Oncol. 2020;21(8):1013-6.

3. O'Donnell JS, Hoefsmit EP, Smyth MJ, Blank CU, Teng MWL. The promise of neoadjuvant immunotherapy and surgery for cancer treatment. Clin Cancer Res. 2019;25(19):5743-51.

4. Keung EZ, Ukponmwan EU, Cogdill AP, Wargo JA. The rationale and emerging use of neoadjuvant immune checkpoint blockade for solid malignancies. Ann Surg Oncol. 2018;25(7):1814-27.

5. Topalian SL, Taube JM, Pardoll DM. Neoadjuvant checkpoint blockade for cancer immunotherapy. Science. 2020;367(6477):eaax082.

6. Versluis JM, Long GV, Blank CU. Learning from clinical trials of neoadjuvant checkpoint blockade. Nat Med. 2020;26(4):475-84.

7. Thomas D, Bello DM. Adjuvant immunotherapy for melanoma. J Surg Oncol. 2021;123(3):789-97.

8. Menzies AM, Scolyer RA, Long GV. Neoadjuvant immunotherapy in melanoma-the new frontier. Clin Cancer Res. 2021;27:4133-5.

9. https://pharmaintelligence.informa.com/products-and-services/dataand-analysis/trialtrove. Accessed 16 Aug 2021.

10. U.S. Food and Drug Administration. Clinical trial endpoints for the approval of cancer drugs and biologics: guidance for industry. December, 2018. https://www.fda.gov/media/71195/download. Accessed 15 Nov 2021

11. Siegel RL, Miller KD, Jemal A. Cancer statistics, 2018. CA Cancer J Clin. 2018;68(1):7-30

12. Zeng $\mathrm{H}$, Chen $\mathrm{W}$, Zheng $\mathrm{R}$, et al. Changing cancer survival in China during 2003-15: a pooled analysis of 17 population-based cancer registries. Lancet Glob Health. 2018;6:e555-67.

\section{Publisher's Note}

Springer Nature remains neutral with regard to jurisdictional claims in published maps and institutional affiliations.

Ready to submit your research? Choose BMC and benefit from:

- fast, convenient online submission

- thorough peer review by experienced researchers in your field

- rapid publication on acceptance

- support for research data, including large and complex data types

- gold Open Access which fosters wider collaboration and increased citations

- maximum visibility for your research: over $100 \mathrm{M}$ website views per year

At BMC, research is always in progress.

Learn more biomedcentral.com/submissions 